\title{
Epistasis: what it means, what it doesn't mean, and statistical methods to detect it in humans
}

\author{
Heather J. Cordell* \\ University of Cambridge, Department of Medical Genetics, JDRF/WT Diabetes and Inflammation Laboratory, \\ Cambridge Institute for Medical Research (CIMR), Wellcome Trust/MRC Building, Addenbrooke's Hospital, \\ Hills Road, Cambridge, CB2 2XY, UK
}

Received July 8, 2002; Accepted July 27, 2002

Epistasis, the interaction between genes, is a topic of current interest in molecular and quantitative genetics. A large amount of research has been devoted to the detection and investigation of epistatic interactions. However, there has been much confusion in the literature over definitions and interpretations of epistasis. In this review, we provide a historical background to the study of epistatic interaction effects and point out the differences between a number of commonly used definitions of epistasis. A brief survey of some methods for detecting epistasis in humans is given. We note that the degree to which statistical tests of epistasis can elucidate underlying biological interactions may be more limited than previously assumed.

Epistasis, defined generally as the interaction between different genes, has become a hot topic in complex disease genetics in recent years. For complex traits such as diabetes, asthma, hypertension and multiple sclerosis, the search for susceptibility loci has, to date, been less successful than for simple Mendelian disorders. This is probably due to complicating factors such as an increased number of contributing loci and susceptibility alleles, incomplete penetrance, and contributing environmental effects. The presence of epistasis is a particular cause for concern, since, if the effect of one locus is altered or masked by effects at another locus, power to detect the first locus is likely to be reduced and elucidation of the joint effects at the two loci will be hindered by their interaction. If more than two loci are involved, the situation is likely to be further complicated by the possibility of complex multiway interactions among some or all of the contributing loci.

In the literature, discussion of epistasis has been considerably confused by differing (usually unstated) definitions and assumptions and by the use of the same terminology to apply to quite different statistical and biological concepts. In essence, epistasis refers to departure from 'independence' of the effects of different genetic loci in the way that they combine to cause disease. However, what is meant by 'independence' is not always precisely stated, and indeed may vary, particularly between the definitions assumed by biologists, epidemiologists, statisticians, and human and quantitative geneticists.

\section{DEFINITIONS OF EPISTASIS}

The term 'epistatic' was first used in 1909 by Bateson (1) to describe a masking effect whereby a variant or allele at one
Table 1. Example of phenotypes (e.g. hair colour) obtained from different genotypes at two loci interacting epistatically, under Bateson's (1909) definition of epistasis

\begin{tabular}{llll}
\hline & \multicolumn{4}{l}{ Genotype at locus G } \\
Genotype at locus B & $g / g$ & $g / G$ & $G / G$ \\
\hline$b / b$ & White & Grey & Grey \\
$b / B$ & Black & Grey & Grey \\
$B / B$ & Black & Grey & Grey \\
\hline
\end{tabular}

locus (denoted at that time as an 'allelomorphic pair') prevents the variant at another locus from manifesting its effect. This was seen as an extension of the concept of dominance for alleles within the same allelomorphic pair i.e. at a single locus. Suppose we have two loci, B and G, that influence a trait such as hair colour in mice. Locus B has two possible alleles, $B$ or $b$, and locus $\mathrm{G}$ has two possible alleles, $G$ or $g$. The possible phenotypic outcomes (white, black or grey hair) given genotype are as shown in Table 1. We see that, regardless of genotype at locus $\mathrm{B}$, individuals with any copies of the $G$ allele have grey hair, i.e., at locus $\mathrm{G}$, allele $G$ is dominant to allele $g$, effectively masking any 'effect' of allele $g$. We also see that if the genotype at locus $\mathrm{G}$ is $g / g$ then an individual with any copies of the $B$ allele has black hair, so that at locus $\mathrm{B}$, allele $B$ is dominant to $b$. If, however, the genotype at locus $\mathrm{G}$ is not $\mathrm{g} / \mathrm{g}$ then the effect at locus B is not observable, since individuals with any copies of the $G$ allele have grey hair regardless of genotype at locus B, i.e. the effect at locus B is masked by that of locus G: locus $G$ is said to be epistatic to locus B (or, more specifically, allele $G$ at locus $\mathrm{G}$ is epistatic to allele $B$ at locus B). 
Table 2. Example of penetrance table for two loci interacting epistatically in a general sense

\begin{tabular}{llll}
\hline & \multicolumn{3}{l}{ Genotype at locus B } \\
Genotype at locus A & $b / b$ & $b / B$ & $B / B$ \\
\hline$a / a$ & 0 & 0 & 0 \\
$a / A$ & 0 & 1 & 1 \\
$A / A$ & 0 & 1 & 1 \\
\hline
\end{tabular}

This definition of epistasis is similar to the concept most often used by a biologist or biochemist when investigating biological interaction between proteins. In fact, what is meant by biological interaction is not always precisely defined (2), but usually corresponds to a situation in which the qualitative nature of the mechanism of action of a factor is affected by the presence or absence of the other (3). There are, however, some problems with this definition, particularly when applied to binary traits. In human genetics, the phenotype of interest is often qualitative and usually dichotomous, indicating presence or absence of disease. Mathematical models for the joint action of two or more loci usually focus on the penetrance, the probability of developing disease given genotype. Suppose that we have two loci, A and B, that influence a binary trait. Locus A has two possible alleles, $A$ or $a$, and locus $\mathrm{B}$ has two possible alleles, $B$ or $b$. Suppose that a predisposing allele is required at both loci in order to exhibit the trait, i.e. one or more copies of both allele $A$ and allele $B$ are required. Then, when the effects of both loci are considered, we obtain the penetrance table shown in Table 2. In this table, the effect of allele $A$ can only be observed when allele $B$ is also present: without the presence of $B$, the effect of $A$ is not observable. The effect at locus A would appear to be 'masked' by that at locus B. By analogy with the example in Table 1, we might say that locus B is epistatic to locus $\mathrm{A}$, since when the genotype $b / b$ is present at locus $\mathrm{B}$, the effect of the alleles at locus A is not observable. However, we might equally well say that locus A is epistatic to locus B, since when the genotype $a / a$ is present at locus A, the effect of the alleles at locus B is not observable. This leads to a situation that is not precisely analogous to that described by Bateson (1). In Bateson's (1) definition, it is clear that if factor B is epistatic to factor A, we do not expect factor A to also be epistatic to factor B. This is illustrated by the lack of symmetry in Table 1 . In Table 2, the symmetry between the effects at loci A and B means that we cannot say that one of the loci is epistatic to the other. Nevertheless this type of penetrance table has been interpreted as representing a more general form of epistasis between the loci (4), albeit of a rather different nature from that originally implied by the term.

Lack of epistasis has classically been represented by penetrance tables such as Table 3 (4-6). This table represents a 'heterogeneity model' in which an individual becomes affected through possessing a predisposing genotype at either locus A or locus B. This would appear to represent a different biological phenomenon to that represented by Table 2 . Table 3 is usually assumed to correspond to a situation in which the biological pathways involved in disease influenced by the two loci are at some level separate or independent (5). However, it is worth noting that if we define the 'effect' of locus B to be a
Table 3. Example of penetrance table for two loci acting together in a heterogeneity model

\begin{tabular}{llcc}
\hline & \multicolumn{3}{l}{ Genotype at locus B } \\
Genotype at locus A & $b / b$ & $b / B$ & $B / B$ \\
\hline$a / a$ & 0 & 0 & 1 \\
$a / A$ & 0 & 0 & 1 \\
$A / A$ & 1 & 1 & 1 \\
\hline
\end{tabular}

recessive disease model (so that two copies of allele $B$ are required to cause disease) then having two copies of allele $A$ at locus A is sufficient to 'mask' this effect, i.e., given genotype $A / A$ at locus $\mathrm{A}$, the effect at locus $\mathrm{B}$ is not observable: locus $\mathrm{B}$ acts differently when the genotype at locus $\mathrm{A}$ is $A / A$ compared with when the genotype at locus $\mathrm{A}$ is not $A / A$. Thus the classical heterogeneity model falls within a definition interpretable as epistasis! This illustrates the inherent problems with biologically motivated definitions of epistasis, unless precise definitions of what is meant by the 'effect' of a locus (or of an allele or genotype at a locus) and 'independence of effects' are given.

The situation has been confused further by the fact that in quantitative genetics, following a paper by Fisher in 1918 (7), the term 'epistatic' has been generally used in yet another different sense from its original usage. In Fisher's 1918 definition, epistasis refers to a deviation from additivity in the effect of alleles at different loci with respect to their contribution to a quantitative phenotype. This definition is not equivalent to Bateson's 1909 definition, as was pointed out in the initial review of Fisher's 1918 paper by R.C. Punnett (8). Epistasis in the Fisher (7) sense is closer to the usual concept of statistical interaction: departure from a specific linear model describing the relationship between predictive factors (here assumed to be alleles at different genetic loci). With this definition, the choice of scale becomes important, since factors that are additive with respective to an outcome measured on one scale may not be additive, i.e. may exhibit interaction when a different, transformed scale is used $(2,9)$.

\section{Mathematical definitions}

Mathematically, the quantitative genetic concept of epistasis may be represented (10) for two loci by the linear model

$$
\begin{aligned}
y= & \mu+a_{1} x_{1}+d_{1} z_{1}+a_{2} x_{2}+d_{2} z_{2}+i_{a a} x_{1} x_{2} \\
& +i_{a d} x_{1} z_{2}+i_{d a} z_{1} x_{2}+i_{d d} z_{1} z_{2},
\end{aligned}
$$

where $y$ is a quantitative phenotype and $x_{i}$ and $z_{i}$ are dummy variables related to the underlying genotype at locus $i$. For example, for a diallelic locus with alleles denoted 1 and 2, we might set $x_{i}=1$ and $z_{i}=-0.5$ for a 1/1 homozygote, $x_{i}=0$ and $z_{i}=0.5$ for a heterozygote, and $x_{i}=-1$ and $z_{i}=-0.5$ for a $2 / 2$ homozygote. The coefficients $\mu, a_{1}, d_{1}, a_{2}$ and $d_{2}$ represent genetic parameters that may be estimated corresponding to the mean effect and additive and dominance effects at the two loci; $i_{a a}, i_{d a}, i_{d a}$ and $i_{d d}$ correspond to epistatic interaction effects. Lack of epistasis in this model implies that all 
interaction coefficients are zero. If there is no epistasis then the resulting model

$$
y=\mu+a_{1} x_{1}+d_{1} z_{1}+a_{2} x_{2}+d_{2} z_{2}
$$

is said to be additive, since, after some algebra, it may be written equivalently as

$$
y_{i j}=\alpha_{i}+\beta_{j},
$$

where $\alpha_{i}$ and $\beta_{j}$ are parameters representing the effects of the genotype $i$ at locus 1 and genotype $j$ at locus 2 , respectively (where $i$ and $j$ take values $0,1,2$ according to the number of copies of the 2 allele present at the locus).

For binary traits, similar models may be applied, but with the outcome of interest usually defined to be $p_{i j}$, the penetrance for genotype $i$ at locus 1 and $j$ at locus 2 . Three models commonly considered are an additive model (5) in which $p_{i j}$ may be written

$$
p_{i j}=\alpha_{i}+\beta_{j},
$$

a multiplicative model $(5,11)$ in which $p_{i j}$ may be written

$$
p_{i j}=\alpha_{i} \beta_{j}
$$

and a heterogeneity model $(4,5)$ in which $p_{i j}$ may be written

$$
p_{i j}=\alpha_{i}+\beta_{j}-\alpha_{i} \beta_{j}
$$

The multiplicative model is usually considered to be an epistatic model in which the loci and pathways involved in disease and influenced by the two loci are not independent. The additive and heterogeneity models are usually assumed to represent non-epistatic models and to correspond to a situation in which the biological pathways are at some level separate or independent. Indeed, the classical heterogeneity model shown in Table 3, whereby an individual becomes affected through possessing a predisposing genotype at either locus 1 or locus 2 , can be formulated as

$$
p_{i j}=\alpha_{i}+\beta_{j}-\alpha_{i} \beta_{j},
$$

with $\alpha_{0}=\alpha_{1}=\beta_{0}=\beta_{1}=0$ and $\alpha_{2}=\beta_{2}=1$. However, the biological interpretation of the heterogeneity model when the penetrances $p_{i j}$ are not constrained to be 0 or 1 is unclear.

The reason that additive and heterogeneity models for the penetrance are often used interchangeably is that it can be shown that these models give very similar results when used to model familial relative risks of disease $(5,12)$. It is not clear, however, that this will hold when modelling other outcomes (e.g. the penetrances themselves in Table 3 cannot be achieved using an additive model). Note that the heterogeneity and multiplicative models can also both be expressed as additive models when transformed to different scales: the heterogeneity model by transforming to a $\log (1-p)$ scale so that

$$
\log \left(1-p_{i j}\right)=\log \left(1-\alpha_{i}\right)+\log \left(1-\beta_{j}\right)=A_{i}+B_{j}, \quad \text { say, }
$$

and the multiplicative model by transforming to a logarithmic scale so that

$$
\log p_{i j}=\log \alpha_{i}+\log \beta_{j}=A_{i}+B_{j}, \quad \text { say. }
$$

In a statistical sense, therefore, all three models can be considered to signify independent additive effects on some appropriate scale. Other models that are additive on specific non-penetrance scales include an additive model for the log odds, popular with epidemiologists,

$$
\log \left(\frac{p_{i j}}{1-p_{i j}}\right)=\alpha_{i}+\beta_{j}
$$

and an additive model for liability on a probit scale (13-16), in which the loci are assumed to contribute to an underlying, unobserved, continuous trait in an additive fashion and development of disease occurs if this trait exceeds a certain threshold. Although these models are additive and therefore expressible without interaction effects on their appropriate scales, they correspond to models with interaction effects (epistasis) when transformed to the penetrance scale.

\section{INTERPRETATION OF EPISTASIS}

With so many conflicting definitions of epistasis, it is not surprising that there has been much confusion in the literature. Indeed, the term epistasis has often been used without being precisely defined, so that it is not clear which definition is being assumed in any given situation. Many authors have assumed that epistasis or interaction between loci refers to departure from additivity on the penetrance scale $(5,17,18)$, whereas others have assumed instead that it refers to a departure from multiplicativity on the penetrance scale (19-21). Moreover, epistasis is sometimes investigated in the context of epistatic variance: the variation of a trait in a population accounted for by epistatic effects (as opposed to simple additive main effects of individual loci). The epistatic variance depends not only on the genetic model for the action of two or more loci, but also on population parameters such as multilocus genotype frequencies $(22,23)$, in the same way that additive and dominance variances at a single locus depend not just on the model of dominance assumed but also on population genotype or allele frequencies.

Confusions of definition and terminology apart, the main problem with the interpretation of epistasis is that the word itself suggests that we are dealing with a biologically interesting phenomenon. If epistasis is detected, the assumption is that this tells us something of interest about the mechanisms and pathways involved in disease - in particular in relation to the biological interaction between implicated proteins. Indeed, the description in (5) hints strongly for a biological or causal interpretation of the models there defined. However, statistical tests of interaction are limited to testing specific hypotheses concerning precisely defined quantities. For statistical testing, we can only focus on mathematical models of epistasis such as those described $(5,7,17)$ rather than those encoded by a rather abstract and vague notion of 'independence' or 'masking' of unspecified 'effects'.

Unfortunately, as we have seen, there is not a precise correspondence between biological models of epistasis and those that are more statistically motivated. We should like to perform a statistical test and interpret the outcome biologically, but this is in general not permissible. Statistical interaction does not necessarily imply interaction on the biological or mechanistic level (24). A brief survey of the epidemiological literature 
reveals the major difficulties that exist in inferring biological meaning from quantitative data measuring disease risk as outcome $(3,25,26)$. The problem is that any given data pattern and statistical model can usually be obtained from a number of completely different underlying mechanisms or models for disease development $(3,26,27)$. For instance, five very different causal mechanisms can be shown to all lead to a multiplicative model for the data used in investigating the joint effects of two risk factors (26). Only if the prior biological model can be postulated in some detail is it likely that statistical modelling of this kind will allow insight into the underlying biological mechanisms.

\section{STATISTICAL METHODS TO DETECT EPISTASIS IN HUMANS}

Since the biological interpretation hinted at in (5) is likely to be unrealistically simplistic, one might ask why we are interested in detecting epistasis in human genetic studies at all? Although the discovery of epistasis may be of limited value for elucidating the underlying biological disease process, allowing for different modes of interaction between potential disease loci can lead to improved power for detection of genetic effects. Simulation studies $(12,28,29)$ suggest that this improvement in power may be relatively modest. Nevertheless, in analysis of real data for type 1 diabetes $(12,28)$, type 2 diabetes (30) and inflammatory bowel disease (31), increased evidence for linkage at one locus was seen when the interaction with another locus was taken into account.

Methods for the detection of epistasis vary according to whether one is performing association or linkage analysis, and according to whether one is dealing with a quantitative or a qualitative (in particular a dichotomous) trait. For genetic association studies, standard methods for epidemiological studies may be employed, with genotypes at the various loci considered as risk factors for disease. Given case-control data genotyped at two diallelic candidate loci, for instance, we may fit the model

$$
\begin{aligned}
\log \left(\frac{p_{i j}}{1-p_{i j}}\right)= & \mu+a_{1} x_{1}+d_{1} z_{1}+a_{2} x_{2}+d_{2} z_{2} \\
& +i_{a a} x_{1} x_{2}+i_{a d} x_{1} z_{2}+i_{d a} z_{1} x_{2}+i_{d d} z_{1} z_{2}
\end{aligned}
$$

(where the genotype variables and coefficients are as defined previously) and compare it with the null model

$$
\log \left(\frac{p_{i j}}{1-p_{i j}}\right)=\mu+a_{1} x_{1}+d_{1} z_{1}+a_{2} x_{2}+d_{2} z_{2}
$$

using standard statistical software packages for logistic regression. This provides an overall 4 degree-of-freedom (df) test for interaction, but the interaction terms could each be tested individually on $1 \mathrm{df}$ by removal from the first model, if required. Note that this procedure implicitly assumes that the log odds scale is the scale of interest: to fit a null model that is additive on the penetrance scale would require use of more specialist statistical software or writing a program to maximize the likelihood directly (27). Quantitative traits can be analysed in a similar way by use of standard multiple linear (as opposed to logistic) regression:

$$
\begin{aligned}
y= & \mu+a_{1} x_{1}+d_{1} z_{1}+a_{2} x_{2}+d_{2} z_{2}+i_{a a} x_{1} x_{2} \\
& +i_{a d} x_{1} z_{2}+i_{d a} z_{1} x_{2}+i_{d d} z_{1} z_{2},
\end{aligned}
$$

where the quantitative phenotype $y$ is the outcome of interest, assumed to be distributed normally given genotype.

Note that these regression procedures are actually designed for testing epistasis between loci that have been genotyped. If it is believed that these loci are not themselves the etiological variants but rather are in linkage disequilibrium (LD) with the true disease-causing variants, then epistasis between the surrogate genotyped loci is likely to be diluted compared with epistasis between the true variants, although the extent to which this occurs will depend on the magnitude of the LD.

A related method for analysis of (nuclear) family data involves a generalization of the genotype relative risk approach proposed by Schaid (32). As originally proposed, given genotype data on a trio consisting of an affected individual plus their parents, this method generates three 'pseudocontrols' consisting of the three alternative genotypes that could have been transmitted to the case, and then analyses them in a matched case-control design. Conditional logistic regression is used to fit models for the genotype relative risks. This method can be extended to fit models for genotype relative risks at two unlinked loci by generating not three but fifteen matched pseudocontrols for each case, where the genotype at the two loci for each pseudocontrol consists of one of the two-locus genotypes that could have been, but was not, transmitted to the case. Two-locus models for the genotype relative risks at the two loci are fitted using conditional logistic regression. Standard statistical software can be used to fit models that involve departure from multiplicativity in the penetrances (and hence in the genotype relative risks); more specialist software or user programming will be required for detecting epistasis defined as departure from additivity in the penetrances. A simpler approach that allows detection of epistatic (but not main) effects is to perform the original 'three-pseudocontrol' analysis (32) at one locus while considering genotype at the other locus to be a covariate that interacts with the first locus. Alternatively, one could use a 'case-only design' in which the distribution of genotypes in affected individuals only is used (33). This requires that genotypes at the loci under consideration be independent of each other in the general population, and, unlike the 'case-pseudocontrol' methods, is not robust to confounding causes of association such as population stratification. A variety of related approaches that focus on the issue of association testing but can be used to detect or allow for epistasis in family-based analysis of quantitative traits have also been proposed (34-37).

Epistasis is relatively easily incorporated into standard nonparametric (model-free) methods of linkage analysis for quantitative traits. One popular method is the variance components method, in which the phenotypic covariance between relatives is modelled in terms of variance component parameters and underlying identity-by-descent (IBD) sharing probabilities at one or more genetic loci, assuming underlying multivariate normality of the trait within pedigrees. Models that include epistatic (in the sense of departure from additive) 
components of variance may be fitted and compared with models that do not contain these components using maximum-likelihood methods implemented in such programs as SOLAR (38). Another popular method of linkage analysis for quantitative traits is the Haseman-Elston method (39) and extensions (40). In this method, the squared difference or product of the phenotype values for a pair of relatives is modelled in a regression framework as a function of the underlying IBD sharing probabilities. To include epistatic interactions between loci, all that is required is to include products of the IBD sharing probabilities at different loci as predictors in the regression equation (40).

For dichotomous traits, model-free methods of linkage analysis typically focus on calculating the likelihood of the observed IBD sharing among pairs of affected relatives. Likelihoods for the IBD sharing at two or more loci can be calculated $(12,28,41)$ under restricted models such as additive, multiplicative or heterogeneity models for the penetrances, and compared with general unrestricted models for the IBD sharing. In this way, tests for epistasis (defined as departure from any of these restricted models) can be performed for either linked $(28,42)$ or unlinked loci. Another approach to the detection of loci that act epistatically is to consider the correlation between IBD sharing or linkage statistics at unlinked loci (30). A correlation of zero is expected under a multiplicative penetrance model, negative correlations provide evidence for a genetic heterogeneity model, and positive correlations suggest the presence of positive epistasis in the sense of departure from a multiplicative model. Tests of linkage that allow for epistasis between the test locus and a previously determined or candidate locus may also be performed $(28,30)$. For full details of all the methods mentioned here, and software availability, the reader is directed towards the relevant references.

\section{CONCLUSIONS}

Methods of analysis that allow for or exploit the phenomenon of epistasis are clearly of growing importance in the genetic dissection of complex disease. A variety of methods exist to detect or control for the presence of epistasis. By allowing for epistatic interactions between potential disease loci, we may succeed in identifying genetic variants that might otherwise have remained undetected. In addition, identification of the most parsimonious statistical model for the joint effects of several loci, including interactions, can be useful for prediction of phenotype and for targeting of interventions. Nevertheless, we have seen that direct biological inference from the results of statistical tests is very difficult. The degree to which statistical modelling can elucidate the underlying biological mechanisms is likely to be limited, and may require prior knowledge of the underlying aetiology. The question of true biological interaction remains of paramount interest, but may ultimately be better answered via molecular, rather than statistical, investigation.

\section{ACKNOWLEDGEMENTS}

The author would like to express thanks to colleagues at Cambridge and Case Western Reserve Universities for a number of useful discussions concerning the origins and definitions of epistasis. The author is supported by a Wellcome Trust Research Career Development Award, funded jointly by the Wellcome Trust and the Juvenile Diabetes Research Foundation.

\section{REFERENCES}

1. Bateson, W. (1909) Mendel's Principles of Heredity. Cambridge University Press, Cambridge.

2. Greenland, S. and Rothman, K.J. (1998) Concepts of interaction. In Winters, R. and O' Connor E. (eds), Modern Epidemiology, 2nd edn. Lippincott-Raven, Philadelphia, pp. 329-342.

3. Siemiatycki, J. and Thomas, D.C. (1981) Biological models and statistical interactions: an example from multistage carcinogenesis. Int. J. Epidemiol., 10, 383-387.

4. Neuman, R.J. and Rice, J.P. (1992) Two-locus models of disease. Genet. Epidemiol., 9, 347-365.

5. Risch, N. (1990) Linkage strategies for genetically complex traits. I. Multilocus models. Am. J. Hum. Genet., 46, 222-228.

6. Schork, N.J., Boehnke, M., Terwilliger, J.D. and Ott, J. (1993) Twotrait-locus linkage analysis: a powerful strategy for mapping complex genetic traits. Am. J. Hum. Genet., 53, 1127-1136.

7. Fisher, R.A. (1918) The correlation between relatives on the supposition of Mendelian inheritance. Trans. R. Soc. Edin., 52, 399-433.

8. Norton, B. and Pearson, E.S. (1976) A note on the background to and refereeing of R.A. Fisher's 1918 paper 'The correlation between relatives on the supposition of Mendelian inheritance'. Notes Rec. R. Soc. Lond., 31 151-162.

9. Frankel, W.N. and Schork, N.J. (1996) Who's afraid of epistasis? Nat. Genet., 14, 371-373.

10. Cockerham, C.C. and Zeng, Z.-B. (1996) Design III with marker loci. Genetics, 143, 1437-1456.

11. Hodge, S. (1981) Some epistatic two-locus models of disease. I. Relative risks and identity by descent distributions in affected sib pairs. Am. J. Hum. Genet., 33, 382-395.

12. Cordell, H.J., Todd, J.A., Bennett, S.T., Kawagushi, Y. and Farrall, M. (1995) Two-locus maximum lod score analysis of a multifactorial trait: joint consideration of IDDM2 and IDDM4 with IDDMI in type 1 diabetes. Am. J. Hum. Genet., 57, 920-934.

13. Fisher, R.A. (1930) The Genetical Theory of Natural Selection. Oxford University Press, Oxford, p.111.

14. Pearson, K. (1900) Mathematical contributions to the theory of evolution VIII: On the inheritance of characters not capable of exact quantitative measurement. Phil. Trans. R. Soc. Lond. A, 195, 79-150.

15. Wright, S. (1934) An analysis of variability in number of digits in an inbred strain of guinea pigs. Genetics, 19, 506-536.

16. Wright, S. (1934) The results of crosses between inbred strains of guinea pigs, differing in number of digits. Genetics, 19, 537-551.

17. Risch, N., Ghosh, S. and Todd, J.A. (1993) Statistical evaluation of multiple locus linkage data in experimental species and relevance to human studies: application to murine and human IDDM. Am. J. Hum. Genet., 53, 702-714.

18. Tiwari H. and Elston R.C. (1998) Restrictions on components of variance for epistatic models. Theor. Popul. Biol., 54, 161-174.

19. Dizier, M.H. and Clerget-Darpoux, F. (1986) Two-disease locus model: sib pair method using information on both HLA and Gm. Genet. Epidemiol., 3, 343-356.

20. Morris, A. and Whittaker, J. (1999) Generalization of the extended transmission disequilibrium test to two unlinked disease loci. Genet. Epidemiol, 17(Suppl 1), S661-S666.

21. Wilson, S.R. (2001) Epistasis and its possible effects on transmission disequilibrium tests. Ann. Hum. Genet., 62, 565-575.

22. Cheverud, J.M. and Routman E.J. (1995) Epistasis and its contribution to genetic variance components. Genetics, 139, 1455-1461.

23. Culverhouse R., Suarez, B., Lin, T. and Reich, T. (2002) A perspective on epistasis: limits of models displaying no main effect. Am. J. Hum. Genet., 70, 461-471.

24. Witte, J. (1998) Gene-environment interaction. In Armitage, P. and Colton, T. (eds), Encyclopedia of Biostatistics. Wiley, Chichester, pp. 1613-1614. 
25. Rothman, K.J., Greenland, S. and Walker, A.M. (1980) Concepts of interaction. Am. J. Epidemiol., 112, 467-470.

26. Thompson, W.D. (1991) Effect modification and the limits of biological inference from epidemiologic data. J. Clin. Epidemiol., 44, 221-232.

27. Cordell, H.J., Todd, J.A., Hill, N.J., Lord, C.J., Lyons, P.A., Peterson, L.B., Wicker, L.S. and Clayton, D.G. (2001) Statistical modeling of interlocus interactions in a complex disease: rejection of the multiplicative model of epistasis in type 1 diabetes. Genetics, 158, 357-367.

28. Cordell, H.J., Wedig, G.C., Jacobs, K.B. and Elston, R.C. (2000) Multilocus linkage tests based on affected relative pairs. Am. J. Hum. Genet., 66, 1273-1286.

29. Leal, S.M. and Ott, J. (2000) Effects of stratification in the analysis of affected-sib-pair data: benefits and costs. Am. J. Hum. Genet., 66 , $567-575$.

30. Cox, N.J., Frigge, M., Nicolae, D.L., Concannon, P., Hanis, C.L., Bell, G.I. and Kong, A. (1999) Loci on chromosomes 2 (NIDDM1) and 15 interact to increase susceptibility to diabetes in Mexican Americans. Nat. Genet., 21, 213-215.

31. Cho, J.H., Nicolae, D.L., Gold, L.H., Fields, C.T., LaBuda, M.C., Rohal, P.M., Pickles, M.R., Qin, L., Fu, Y., Mann, J.S. et al. (1998) Identification of novel susceptibility loci for inflammatory bowel disease on chromosomes 1p, 3q, and 4q: evidence for epistasis between $1 \mathrm{p}$ and IBD1. Proc. Natl Acad. Sci. USA, 95, 7502-7507.

32. Schaid, D.J. (1996) General score tests for associations of genetic markers with disease using cases and their parents. Genet. Epidemiol., 13, 423-449.
33. Umbach, D.M. and Weinburg, C.R. (1997) Designing and analysing casecontrol studies to exploit independence of genotype and exposure. Statist. Med., 16, 1731-1743.

34. Rabinowitz, D. (1997) A transmission disequilibrium test for quantitative trait loci. Hum. Hered., 47, 342-350.

35. George, V., Tiwari, H., Zhu, X. and Elston, R.C. (1999) A test of transmission/disequilibrium for quantitative traits in pedigree data, by multiple regression. Am. J. Hum. Genet., 65, 236-245.

36. Lunetta, K.L., Faraone, S.V., Biederman, J. and Laird, N. (2000) Familybased tests of association and linkage that use unaffected sibs, covariates, and interactions. Am. J. Hum. Genet., 66, 605-614.

37. Liu, Y., Tritchler, D. and Bull, S.B. (2002) A unified framework for transmission-disequilibrium test analysis of discrete and continuous traits. Genet. Epidemiol., 22, 26-40.

38. Almasy, L. and Blangero, J. (1998) Multipoint quantitative-trait linkage analysis in general pedigrees. Am. J. Hum. Genet., 62, 1198-1211.

39. Haseman, J.K. and Elston, R.C. (1972) The investigation of linkage between a quantitative trait and a marker locus. Behav. Genet., 2, 3-19.

40. Elston, R.C., Buxbaum, S., Jacobs, K.B. and Olson, J.M. (2000) Haseman and Elston revisited. Genet. Epidemiol., 19, 1-17.

41. Olson, J.M. (1999) A general conditional-logistic model for affectedrelative-pair linkage studies. Am. J. Hum. Genet., 65, 1760-1769.

42. Farrall, M. (1997) Affected sibpair tests for multiple linked susceptibility genes. Genet. Epidemiol., 14, 103-115. 